\title{
Modeling Off-Nominal Recovery in NextGen Terminal-Area Operations
}

\author{
Todd J. Callantine* \\ San Jose State University/NASA Ames Research Center, Moffett Field, CA, 94035
}

\begin{abstract}
Robust schedule-based arrival management requires efficient recovery from off-nominal situations. This paper presents research on modeling off-nominal situations and plans for recovering from them using TRAC, a route/airspace design, fast-time simulation, and analysis tool for studying NextGen trajectory-based operations. The paper provides an overview of a schedule-based arrival-management concept and supporting controller tools, then describes TRAC implementations of methods for constructing off-nominal scenarios, generating trajectory options to meet scheduling constraints, and automatically producing recovery plans.
\end{abstract}

\section{Introduction}

A ground-based precision scheduling system and air traffic controller tools to aid in the schedule-based management of aircraft flying optimized profile descents (OPDs) along Area Navigation (RNAV) routes have been developed at NASA Ames Research Center as part of a concept for realizing Next Generation Air Transportation System (NextGen) Air Traffic Management (ATM) initiative objectives ${ }^{1}$ in the terminal area. ${ }^{2,3}$ A recent human-in-the-loop simulation study investigated the robustness of the controller tools to off-nominal situations; a traffic management supervisor updated arrival schedules as required for controllers using the support tools to manage off-nominal events (e.g., go-around's, 'no-radio' ('NORDO') aircraft) scripted to occur during the experimental trials. ${ }^{4}$

The present research complements these efforts by exploring ways to use the Trajectory-Based Route Analysis and Control (TRAC) fast-time simulation tool to model similar off-nominal situations and investigate recovery plans for restoring nominal operations. TRAC is a publically available Java-based tool that includes trajectory modeling, scheduling, and trial-planning functionality useful for examining trajectory-based ATM concepts. ${ }^{5,6}$ This paper presents new TRAC functionality for constructing off-nominal scenarios and generating trajectory options, as well as preliminary research toward a heuristic search process for generating 'recovery plans' that specify an updated schedule along with aircraft trajectories for achieving the new schedule.

The paper begins by providing background on the schedule-based arrival-management concept and supporting controller tools, as well as the human-in-the-loop simulations conducted to examine nominal and off-nominal operations. The background section also describes previously-implemented TRAC functionality that the current work leverages. Subsequent sections then describe functionality for and illustrative examples of, in turn, off-nominal scenario construction, trajectory-option generation, and recovery-plan generation. The paper concludes with a discussion of potential improvements and possible future research directions.

\section{Background}

Managing aircraft on OPDs in dense traffic conditions is inherently difficult because, even if aircraft are set up to arrive properly spaced (e.g., Ref. 7), some adjustments are required to correct for disturbances that accrue during the descent (e.g., due to forecast wind errors, pilotage, etc.) unless excessive buffers are introduced. However, currentday control techniques (i.e., vectoring) interrupt the OPDs. Speed adjustments enable uninterrupted OPDs, but without suitable tools speed adjustments alone are difficult to perform. To address these problems an operational concept for maintaining high-density OPDs has been developed that relies on ground-based scheduling and controller support tools. Aircraft are assumed to have been controlled en route such that they descend into the terminal area with reasonably small runway schedule errors (i.e., $60 \mathrm{~s}$ early to $30 \mathrm{~s}$ late) that can be corrected with

\footnotetext{
* Senior Research Engineer, Human Systems Integration Division, NASA Ames Mail Stop 262-4, AIAA Senior Member.
} 
speed control. As aircraft transit the terminal area along RNAV/OPD routes, controllers use the tools to issue speed adjustments as required to effect merges and achieve schedule conformance such that a last adjustment by the Final controller, if necessary, can ensure each aircraft arrives properly spaced at the runway threshold. Thus, under nominal conditions the integrity of underlying trajectory predictions is maintained by keeping aircraft on their RNAV routes, so that the schedule and trajectory-based tools can adequately support controllers in issuing required speed adjustments-enabling aircraft to remain on their assigned RNAV routes and execute uninterrupted OPDs.

Ref. 3 describes one in a series of 'Controller Managed Spacing' (CMS) human-in-the-loop simulation studies that demonstrated schedule timelines, 'slot marker' circles, and speed advisory tools can effectively support controllers operating under this concept. Timeline displays present, for scheduling points relevant to each controller, the estimated and scheduled times of arrival (ETAs and STAs) of each aircraft (Fig. 1). Slot markers translate the schedule information to a spatial target on the controller's display that shows where each aircraft would be if it were flying its assigned nominal OPD profile and arrived on schedule (Fig. 2). Speed advisories appear when trajectory predictions indicate a speed is available that, if issued, would enable the aircraft to achieve schedule conformance and rejoin the nominal speed profile at a specified point (Fig. 3). Feeder controllers issued speeds to get aircraft as close to their slot markers as possible before transferring control to a Final controller, who made additional adjustments and, if necessary, issued a final speed adjustment to ensure the relative spacing of the aircraft on final approach would result in proper spacing at the runway threshold. The results showed that good schedule conformance and proper spacing are achievable for moderately high levels of runway throughput while enabling all aircraft to conduct OPDs and remain on their assigned RNAV routes.

A recent study examined the robustness of the concept to off-nominal situations, in which aircraft suffered radio outages, needed to go around due to runway obstructions or mechanical problems, or requested expedited arrivals due to on-board medical emergencies. These situations were scripted to occur at predetermined points during traffic scenarios such that large schedule errors could result. When go-arounds were reinserted into a schedule, for example, aircraft behind them might need large delays; NORDO aircraft could not receive clearances, and aircraft in front of or behind the NORDO aircraft might be affected depending upon whether the NORDO aircraft was ahead or behind schedule. Controllers had all the CMS tools available, and could also issue prespecified path options (i.e., alternative approach transitions) and specify

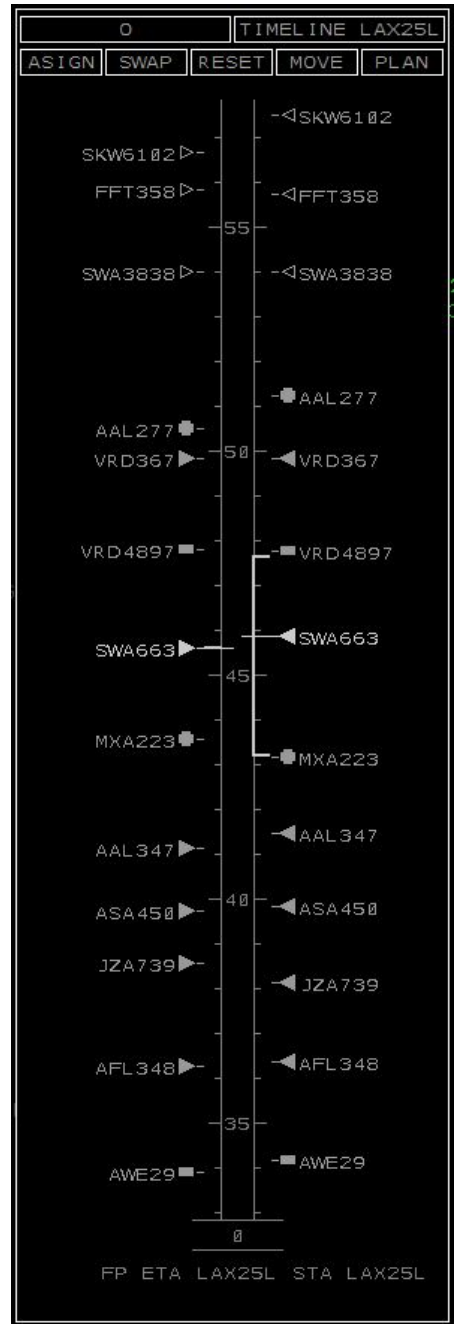

Figure 1. Timeline with ETAs on the left, STAs on the right, and one 'required spacing' bracket visible.

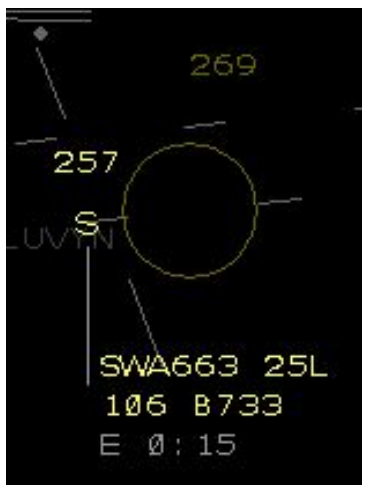

Figure 2. Aircraft with target location outside slot marker.

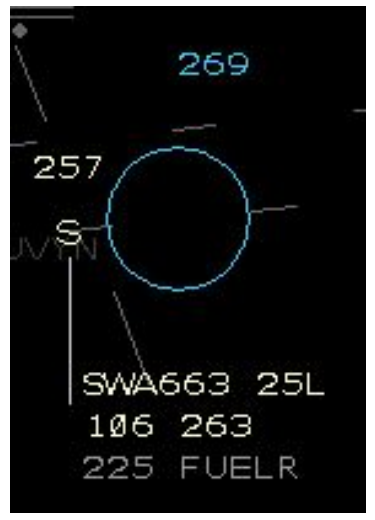

Figure 3. Speed advisory shown in third line of aircraft data block. these paths to the automation, so that aircraft could absorb larger delays than would be possible with speed alone while still enabling the slot markers and speed advisories to work along the modified RNAV routes. The cast of controller participants was also expanded to include a traffic management supervisor responsible for adjusting runway schedules to accommodate offnominal aircraft by reassigning STAs as necessary to produce a workable plan for recovering from offnominal situations. Schedule adjustments included STA-reassignments for individual aircraft, STA swaps, and rescheduling specified blocks of aircraft in the arrival sequence. A key observation from this study is that when the supervisor was able to produce an achievable schedule in a timely manner (e.g., within a minute or two), and the controllers immediately began working toward it, they could recover from an off- 
nominal situation smoothly. On the other hand, if the schedule remained in flux, or had STAs that were difficult to achieve (e.g., because an aircraft had already passed an alternate approach transition's starting point), recovery was more difficult and took longer. It was also observed that, in general, it was more difficult to advance aircraft than to delay them.

The present research explores how fast-time simulation models implemented in TRAC can be used to investigate how to manage similar sorts of off-nominal situations. Three areas of potential functionality were identified: creating the off-nominal situations, generating trajectory options for absorbing large delays, and developing recovery plans for restoring nominal operations. TRAC already includes functionality for generating scenarios from recorded traffic, ${ }^{6}$ and automatically generates arrival traffic with schedule errors generated according to specified distributions (Ref. 8 provides examples of a related technique implemented in a precursor to TRAC). The focus here, however, is on methods for adapting the position or behavior of aircraft in a TRAC simulation such that offnominal situations are created at specific times. TRAC also implements trial-planning functionality that could be leveraged to create off-nominal behaviors. ${ }^{6}$ Trial plans, as well as degree-of-freedom specifications, ${ }^{9}$ also provide a suitable basis for generating trajectory modifications that could be used resolve off-nominal situations. Ref. 9 provides an early example of an off-nominal recovery simulation using path degrees of freedom. Finally, TRAC has extensive scheduling capabilities that can serve as the basis for recovery planning similar to that performed by the traffic management supervisor in the recent simulation. The following sections describe enhancements to these capabilities intended to support fast-time investigations of off-nominal situations.

\section{Off-Nominal Scenario Construction}

The first challenge in modeling off-nominal recovery using fast-time simulations is to simulate off-nominal situations. This section describes three capabilities implemented in TRAC for this purpose. The first is the capability to trial-plan trajectory modifications that yield off-nominal situations, then re-simulate those changes automatically. Because the off-nominal situation is of primary interest, a second capability was developed to capture simulations in progress - at a point at or just before the off-nominal situation arises. Finally, the need to make small adjustments to traffic scenarios exists for all fast-time simulations that use pre-established scenarios (i.e., scenarios that are not randomly constructed at run time). To address this need, a third capability to make spatio-temporal adjustments was also developed.

\section{A. Resimulating Trajectory Modifications}

Figure 4 shows traffic arriving on RNAV routes to runways 24R and 25L at Los Angeles International Airport (LAX). The aircraft are currently set up to arrive at their scheduled times (white schedule entries in Fig. 5). To create an off-nominal situation, trial plans are constructed for two aircraft ('AC013' and 'AC014'). The trial plan for AC013 specifies a speed clearance that causes it to ignore downstream speed restrictions and arrive ahead of schedule; the trial plan for AC014 departs from the nominal RNAV route, as if the aircraft were vectored along that path. Figure 5 shows the adjusted ETAs corresponding to the two trial plans in green.

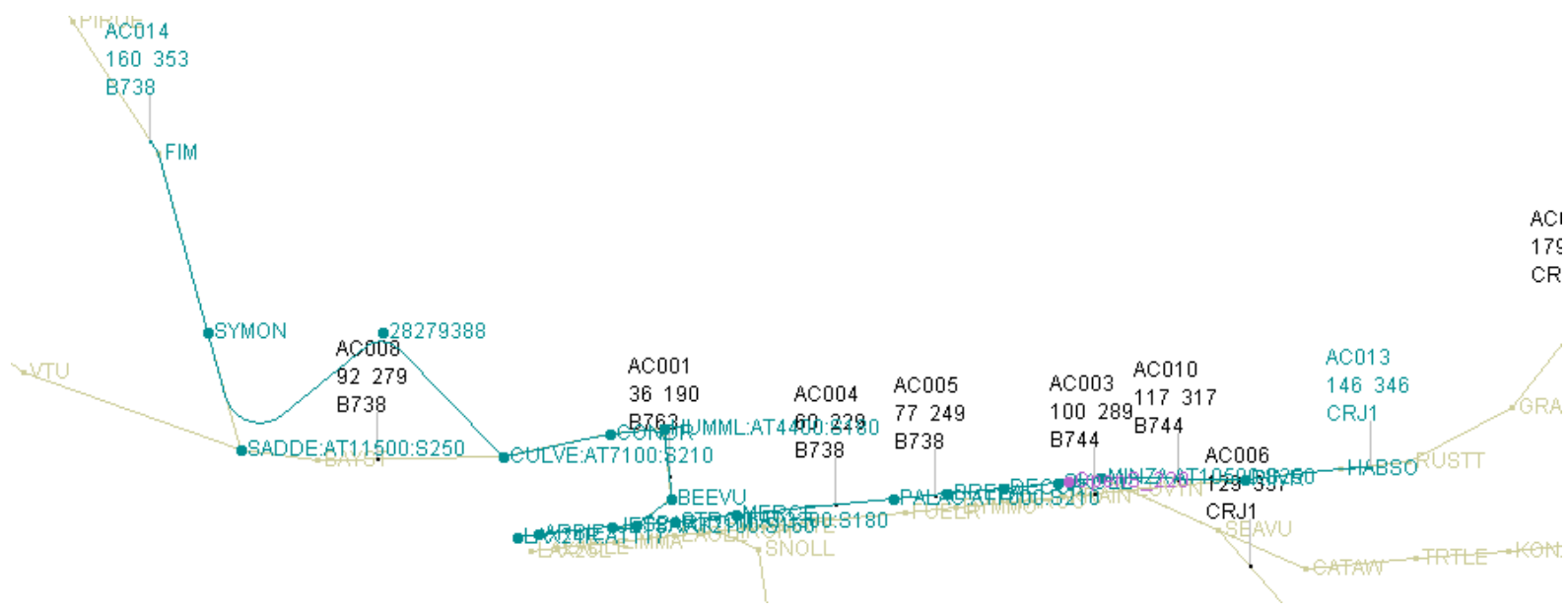

Figure 4. TRAC trial plans constructed for two aircraft in an LAX arrival flow. 
A user may create a new simulation in which the trajectory modifications reflected in the trial plans occur automatically by first executing the two trial plans and allowing the simulation to run to completion. The user may then press a button to create a new simulation configuration which, when run, schedules and automatically executes the trajectory modifications. The new simulation configuration can be saved in a TRAC project file for later use. Figure 6 depicts the traffic situation when the new simulation has run to a time slightly after the stored trajectory modifications were triggered. AC013 is out-of-conformance with respect to the nominal speed along the route, while AC014 has the appearance of being vectored back toward the downwind leg (the 'broadened' downwind leg is a consequence of applying RNAV route design criteria to a continuous RNAV route to the runway). As shown on the timeline in Fig. 7, the schedule now has several aircraft out-ofconformance.

This process affords precise repeatability of trajectory modifications, including multiple modifications for each aircraft, but eliminates the possibility of modifying the aircraft for which trajectory changes occur. All parameters (e.g., aircraft type, initial state and time, winds, etc.) for aircraft with scheduled trajectory modifications must remain the same, lest the modification create a trajectory discontinuity. However, an aircraft's trajectory can be further modified (e.g., via a new trial plan) after the last scheduled trajectory modification has occurred.

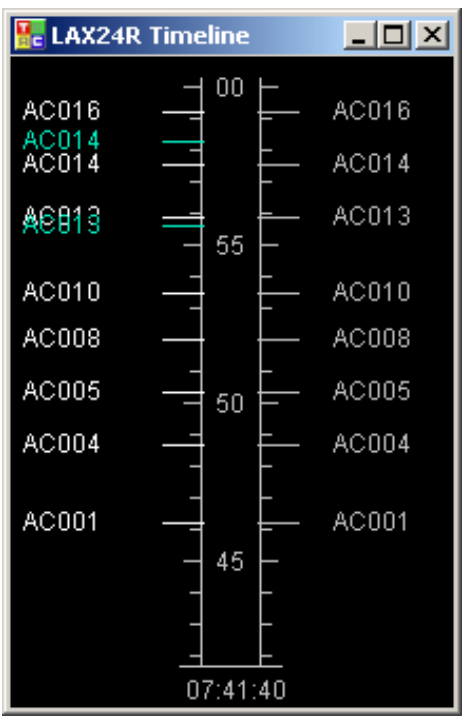

Figure 5. Runway schedule timeline, with trial-plan ETAs shown in green.

\section{B. Capturing Simulations in Progress}

To facilitate examination of off-nominal situations created by resimulating trajectory modifications, the capability to capture a simulation in progress was also implemented in TRAC. A user may create a new simulation that starts with all aircraft in their current states by pausing a simulation and pressing a button. The new simulation can also be saved for later use, but because aircraft trajectories are saved directly, it is again subject to the limitation that users cannot edit aircraft parameters. For this reason, simulation configurations with scheduled trajectory modifications and those captured from a simulation in progress are clearly identified in the title bar of their respective simulation configuration properties windows.

\section{Adjusting Traffic Scenarios}

Once a simulation has run to completion, aircraft and schedule states are available for replay. The present research leverages historic state information to make both spatial and temporal adjustments to aircraft possible, enabling users to directly manipulate aircraft positions and/or their schedule entries to alter traffic scenarios as

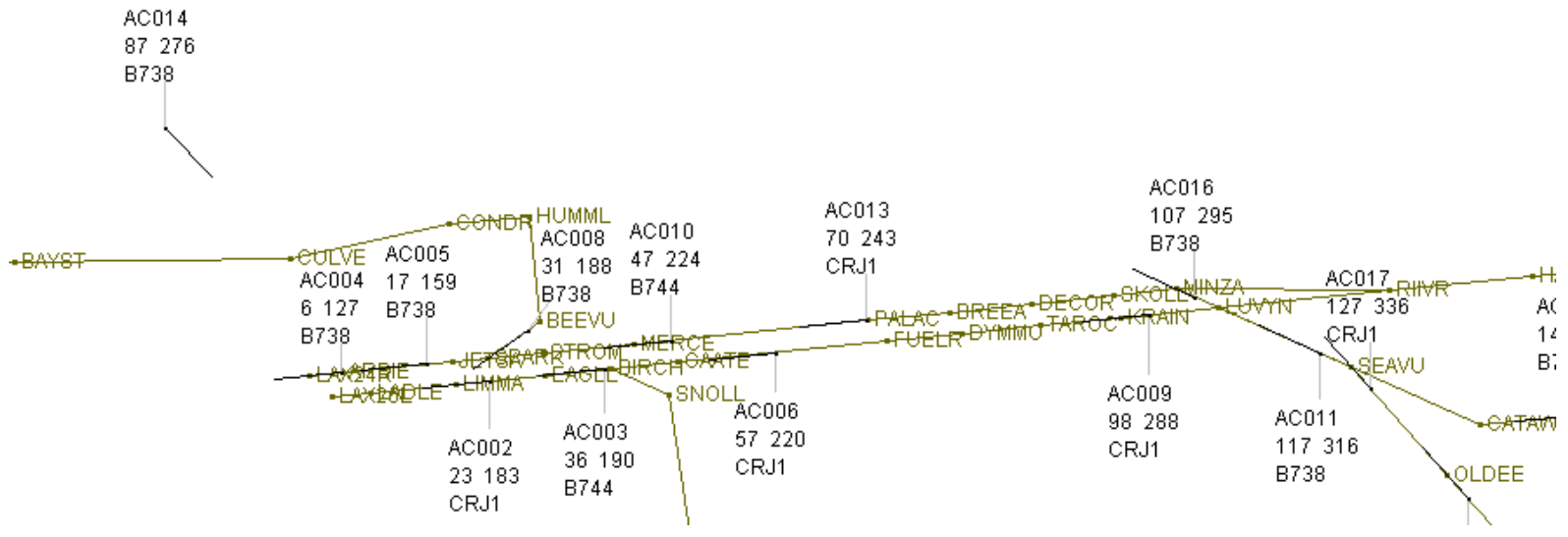

Figure 6. Simulation of scenario with trajectory changes triggered to create an off-nominal situation. 


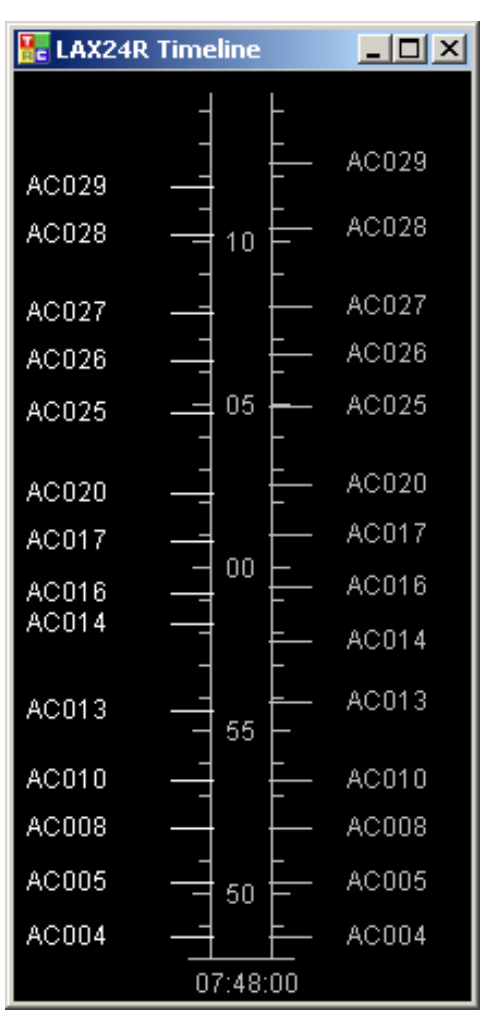

Figure 7. Runway schedule timeline following trajectory modifications.

desired. Adjustments of this sort are useful for creating off-nominal events, as well as adding or removing conflicts, establishing desired schedule errors, or creating specific sector loadings.

When a user chooses to adjust a simulation configuration, TRAC creates 'ghost' aircraft for all the simulation aircraft and the dialog shown in Fig. 8 appears. The left pane of the dialog enables a user to time-shift individual aircraft or groups of selected aircraft by a cumulative or absolute amount of

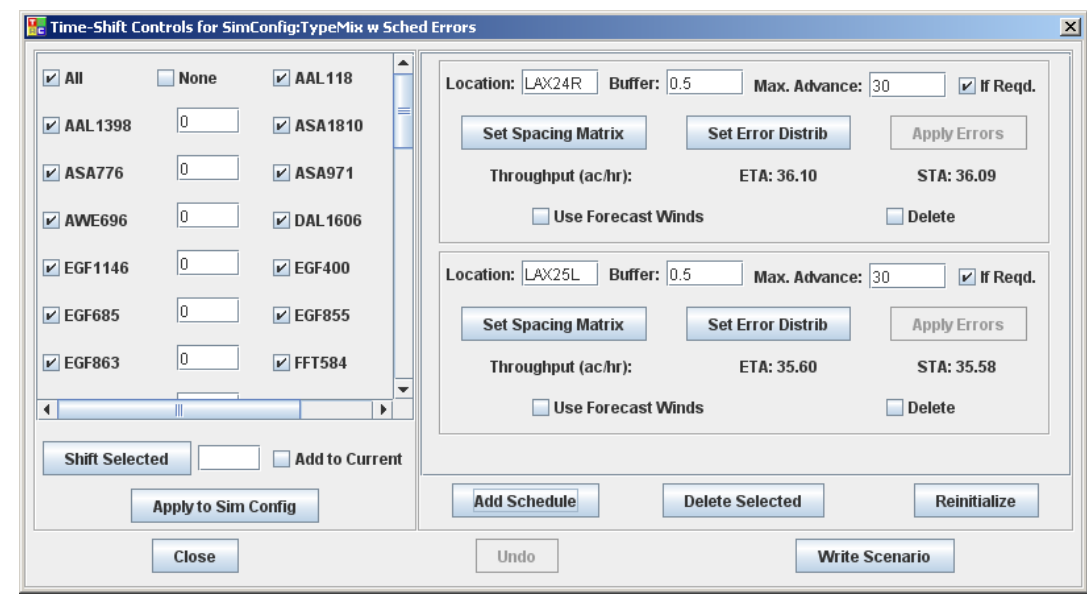

Figure 8. Dialog for setting time-shift values and configuring schedules.

time. The right pane of the dialog enables a user to configure schedules at desired locations. A user may also interact directly with the traffic display or schedule timelines to adjust aircraft. Figure 9 shows how an aircraft's ETA appears as it is being dragged to a new location on the timeline. Schedule STAs are also manipulable and may be frozen or dragged to alternate locations to examine how a schedule changes in response to aircraft adjustments. Figure 10 shows the TRAC planview display, with ghost aircraft (in blue) reflecting the adjusted aircraft positions. A user may also drag aircraft targets along their lateral paths to

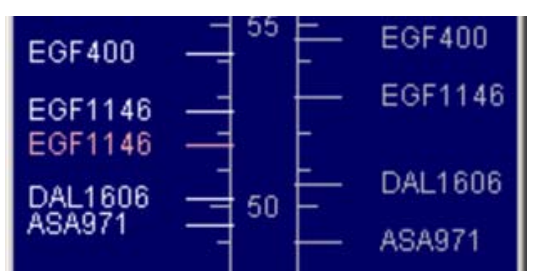

Figure 9. Dragging an ETA on a scenario-adjustment timeline.

effect adjustments. In this example, EGF1146 has been advanced using the timeline after ASA971 and SWA1198 have been repositioned on the traffic display (unadjusted aircraft appear as blue ghost aircraft, as their ghost data block is directly on top of their actual datablock). The time-adjustment control at the bottom of the Fig. 10 enables a user to replay the simulation and examine where aircraft will be at any point in time if the simulation is allowed to run without control interventions.

Any adjustments to the traffic scenario aircraft are reflected in the time-shift control dialog. Once a user has made all the desired adjustments, pressing the 'Apply to Sim Config' button (see Fig. 8) adjusts the simulation configuration to reflect the changes. A user can make further adjustments by re-running the simulation to completion and repeating the adjustment process.

A user may also apply the TRAC traffic scenario-adjustment capability to other simulation data. A user may, for example, load data from (uncontrolled) MACS aircraft ${ }^{10}$ into TRAC and examine how the traffic would appear if certain adjustments were applied. This capability has proved useful in developing traffic scenarios for human-in-theloop simulation studies (e.g., Refs 3 and 4). Taken together, the capabilities to resimulate trajectory changes, capture scenarios, and adjust aircraft by time-shifting them are powerful tools for simulating off-nominal situations repeatably without the need to wait for the situation of interest to arise. The following sections turn to functionality useful for investigating recovery from off-nominal situations created using these capabilities. 


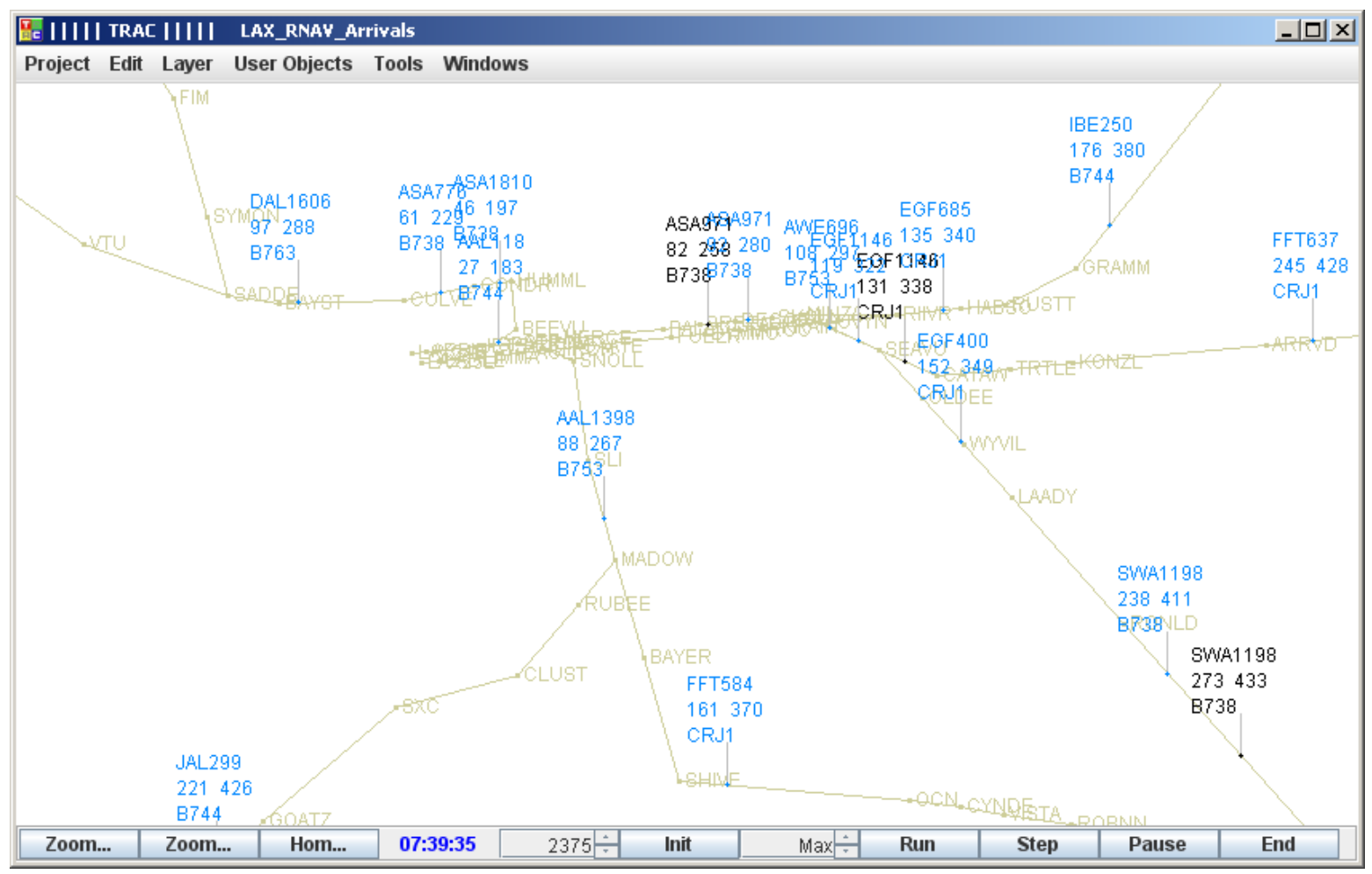

Figure 10. TRAC plan-view display showing three ghost aircraft shifted from their original position (shown in black); all other ghost aircraft remain exactly on top of their associated simulation aircraft.

\section{Trajectory-Option Generation}

To understand how controllers might recover from off-nominal situations under a schedule-based arrival management concept, an understanding about available clearances and their effects on relevant schedules is needed. For a trajectory-based simulation tool such as TRAC, this implies a capability to generate potential recovery trajectories and examine the resulting aircraft ETAs and how they affect the schedules. While the TRAC trialplanning functionality described in Ref. 6 enables a user to freely generate trajectory options, this research seeks automatically generated trajectory options that maintain some correspondence with the types of options available to controllers in the recent human-in-the-loop simulation of off-nominal events. There controllers could, of course, vector aircraft as necessary, but when path adjustments were required the preferred technique was to assign alternative RNAV approach transitions and fine-tune the arrival times using speed clearances.

In the human-in-the-loop study, as with most ATM automation in which path options play a role, the available path options were prespecified and identifiable by name. Such 'adaptation'-based approaches are effective, but have drawbacks related to constructing and maintaining the paths that comprise the adaptation for a particular airspace. In the far term, when arrival routes may be dynamically generated (e.g., Ref. 11), some means for specifying route options for the dynamically generated routes is needed. The TRAC degree-of-freedom specification ${ }^{9}$ goes some way toward addressing this problem, but in off-nominal situations some aircraft may be off-route, so the usefulness of prespecified path degrees of freedom is diminished in some cases.

A more flexible methodology for generating trajectory options was developed and implemented in TRAC in a preliminary form. Data communications are assumed available for communicating selected trajectories to the aircraft (unlike the recent human-in-the-loop study, in which named transitions were communicated by voice). The implementation uses a prioritized scheme, in which TRAC first tries to find a trajectory generated in response to a previous query, followed by speed-change options along the current lateral path, followed by path options, then path options with speed changes. The process returns the first trajectory it encounters that yields an ETA within tolerance. The ETA tolerance is currently set between $-2 \mathrm{~s}$ (two seconds of advance still needed) and $5 \mathrm{~s}$ (five seconds of delay still needed). As each trajectory is generated it is stored in a hash table indexed by the resulting ETA for possible retrieval if it is within tolerance for a subsequent query to the option generator. 
In the current implementation TRAC generates speed-change options along the current lateral path by assuming the speed change will be initiated in one minute. It begins by identifying the slowest speed, the next-slower speed from the current speed, the next-faster speed from the current speed, and the fastest speed, assuming a five-knot speed quantization. The slowest speed is altitude-limited (e.g., $150 \mathrm{kts}$ if the aircraft is below $3000 \mathrm{ft}$; $180 \mathrm{kts}$ below $10,000 \mathrm{ft} ; 190 \mathrm{kts}$ above 10,000 ft). The fastest speed is bounded by $250 \mathrm{kts}$ below 10,000 ft and VMO-minus-10 kts above 10,000 ft. Given these speed values and information about whether the aircraft needs to be advanced or delayed, the speed change algorithm inserts speed change points into a trial-plan trajectory and updates the trajectory until it either finds a within-tolerance ETA, or tests the slowest or fastest speed without success. The algorithm also considers two types of speed clearances: 'open' speeds (i.e., a tactical 'maintain speed' clearance), and 'speed-tonext-lower-charted' speed (in which the aircraft maintains the given speed until it needs to slow to meet a slower speed restriction at a downpath waypoint). If the aircraft needs to be advanced, the algorithm tries the former type first; if it requires delay, the algorithm tries the latter type first.

TRAC generates path options using a raytracing approach, again starting with a location and future course one minute ahead of the aircraft along its current trajectory. It uses information about all the routes used by aircraft currently in the simulation to find route waypoints within 60 degs to the left or right of the starting course and within $60 \mathrm{nmi}$ of the starting location. To maintain flow consistency, rays from the starting location that intersect another route before reaching the given waypoint are discarded. In addition, TRAC includes degree-of-freedom specifications that yield base extensions, so that base-extension paths are included in the set of path options. Figure 11 shows the results of the path-optiongeneration process for aircraft on the LAX RNAV arrival routes. Note that in some cases, the process may yield an option to go to a different runway than the one an aircraft is currently assigned to; this is because runway changes should be considered when developing plans for recovering from off-nominal situations. If none of the path options TRAC generates yield a within-tolerance ETA, the speed search procedure is used along optional paths. If the aircraft requires delay, the next longer path is used; if the aircraft needs to be advanced, the next shorter path is used-until all the suitable paths are exhausted. Again, each trajectory that TRAC generates is stored for
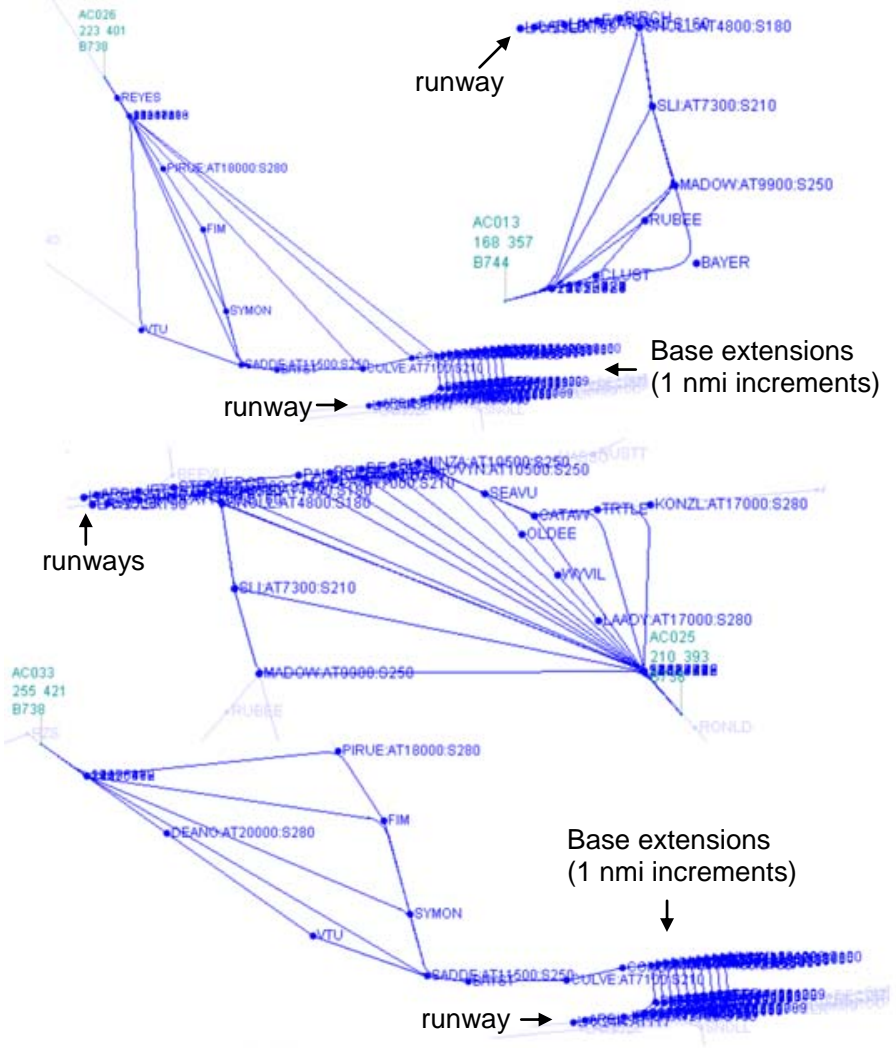

Figure 11. Examples of path options generated by TRAC. possible retrieval in response to future queries.

A menu accessible from an aircraft's trial plan enables a user to exercise the trajectory option-generation procedure via the dialog shown in Fig. 12. Figure 13 shows the results of several successful queries for which the trajectory TRAC generates is within the specified tolerance. The results illustrate the sorts of delays that are possible for aircraft arriving on various RNAV routes to LAX. For example, a slow speed issued to a 'straight-in' arrival can be used to absorb $30 \mathrm{~s}$ of delay, while path options (and base extensions in particular) afford considerably more control authority when used in conjunction with a speed

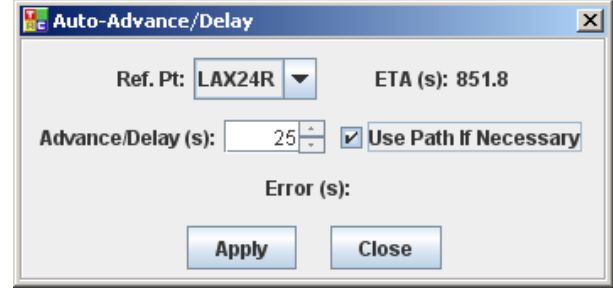

Figure 12. Trajectory-option query dialog. adjustment. 


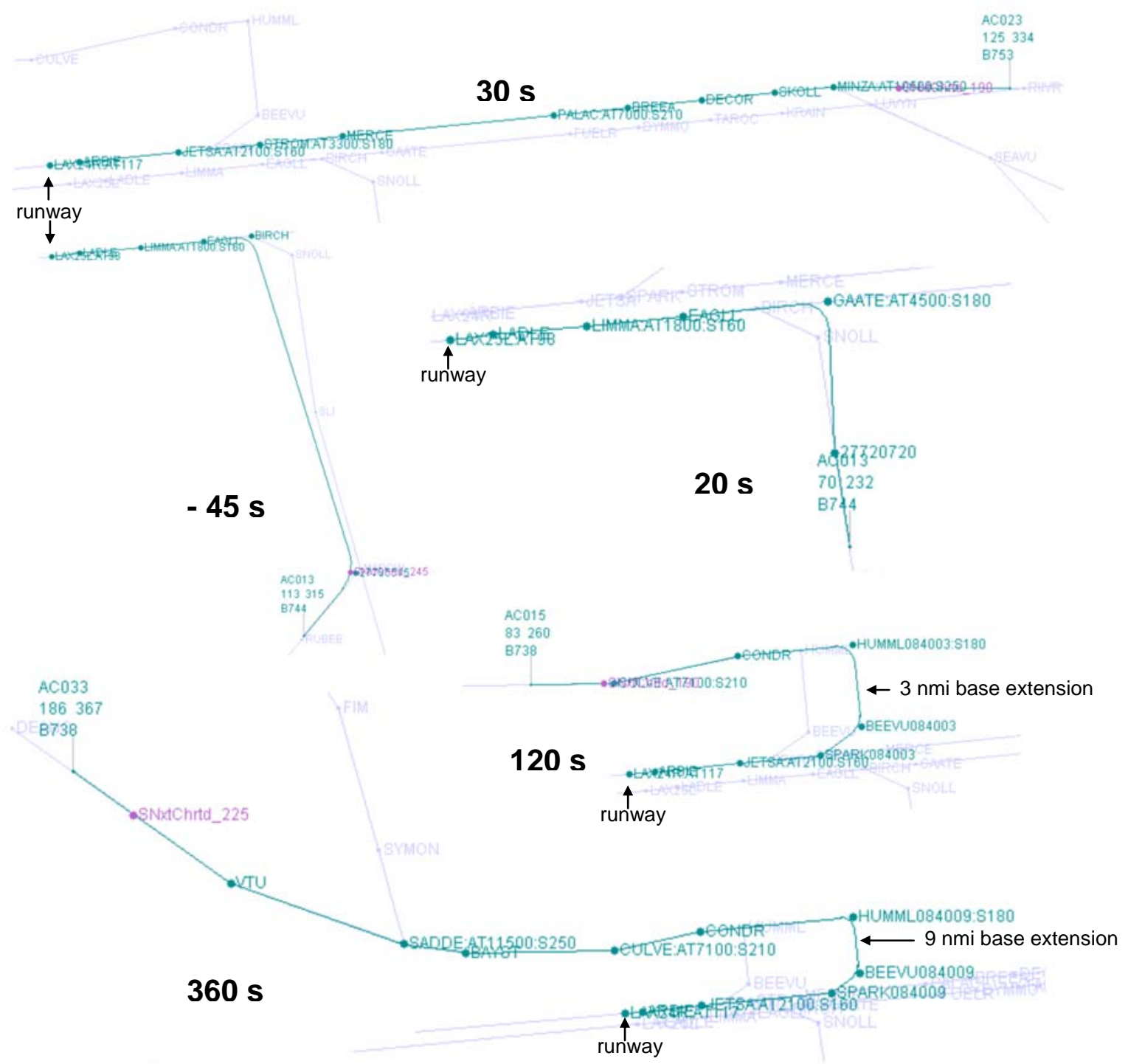

Figure 13. Trajectories generated by TRAC to yield the indicated advance or delay.

In the current implementation, if TRAC cannot generate a within-tolerance trajectory option, it installs the option with the closest smaller ETA (such that the path option still requires some delay). This approach reflects the idea that off-nominal recovery may occur in stages through two or more trajectory adjustments as an aircraft nears the runway. Because advancing an aircraft becomes more difficult as an OPD progresses, trajectories that require delay are preferred.

To limit the search the current implementation currently ignores vectoring options, which may in fact be required to recover from a particular off-nominal situation. Vectoring options are compatible with the current approach, however, and may be generated by specifying quantizations for vectoring angle and leg length, along with a limit on the number of vectors allowed. At each level of the search, each turn to a new course supplies the starting course and location for input to the waypoint-search procedure used to find path options, yielding vectoring legs followed by a direct-to point for rejoining a nominal RNAV route. This process could allow intersections with other paths along vectoring legs, as often occurs during vectoring operations. Tests of an algorithm like this in many cases yielded long processing times, so this functionality was excluded for now based on the idea that multi-stage trajectory modifications are often feasible. 
In general, the prototype implementation could benefit from greater context-specificity with regard to ETA tolerances, speed ranges, clearance distances, and the parameters used to constrain the path-option search. For example, the current ETA tolerances are reasonable for aircraft on final approach, when there may be but a 'last chance' to achieve the required arrival spacing, but could be larger for aircraft just entering the terminal area. In these cases the current implementation may produce an alternative lateral path when it may still be possible to advance the aircraft along the nominal RNAV arrival. Similarly the 60-degree path-option search is principally required to produce path options in 'tight' airspace at low altitudes; it may yield some overly aggressive path changes for aircraft at higher altitudes (and could be even more aggressive at low altitudes). The greedy manner in which trajectories are returned is also disadvantageous in some cases. For example, if TRAC finds a speed near the bottom of an aircraft's speed range that produces a within-tolerance result for the aircraft's current lateral path, that result is returned when a more preferable nominal-speed path adjustment may actually exist. These issues are reserved for future research; the following section describes work that leverages the currently implemented pathoption-generation process to generate recovery plans for off-nominal situations.

\section{Trajectory-Based Off-Nominal Recovery Planning}

Given the apparent importance of timely schedule adjustments for efficiently recovering from off-nominal events, the present research explores methods for producing a 'recovery plan' (i.e., an updated schedule together with trajectories for realizing it) that could at least serve as a starting point. Optimal scheduling is a difficult combinatorial optimization problem that can be formulated as a mixed-integer linear program (MILP) provided the potential arrival times for aircraft are known. As in Ref. 12, this research instead seeks a heuristic method that leverages TRAC's capability to generate trajectory options for achieving required STAs. A candidate method has been implemented in TRAC that enables a user to select a schedule to 'auto-plan,' then visualize the resulting schedule and trajectory modifications. A user may then implement the trajectory modifications if desired. Figure 14 shows examples of arrival schedules for runway 24R and 25L at LAX. A NORDO aircraft ('AC026N') is currently estimated to arrive late according to the LAX24R schedule, while a go-around ('AC005G') has been inserted in the LAX25L schedule. Invoking the auto-planning process on both produces schedules (Fig. 15) and modified trajectories (Fig. 16) that, in this case, enable full recovery from the off-nominals. Two of the nine trajectories produced include path adjustments in addition to speed changes.

The auto-planning algorithm works in a manner similar to the traffic management supervisor in the recent simulation study. It first identifies a 'block' of aircraft in the selected schedule, starting with the first aircraft whose STA-ETA error is out-of-tolerance, and ending with the first aircraft whose STA is not yet frozen. The algorithm first computes the feasible advance/delay range for each aircraft, from the fastest speed along its shortest available path option to the slowest speed along its longest available path option. The STAs of any NORDO aircraft (i.e.,
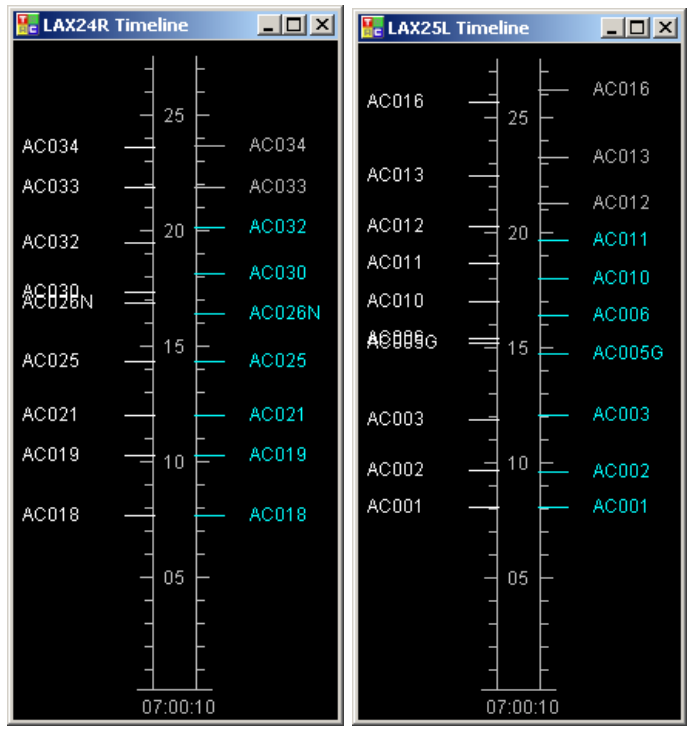

Figure 14. Initial schedules for an off-nominal situation.
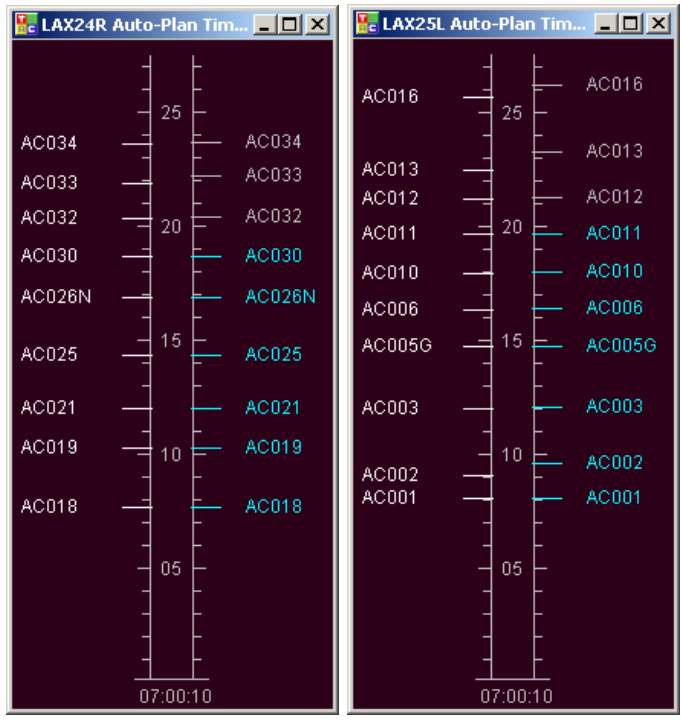

Figure 15. Auto-planned schedules for offnominal recovery. 


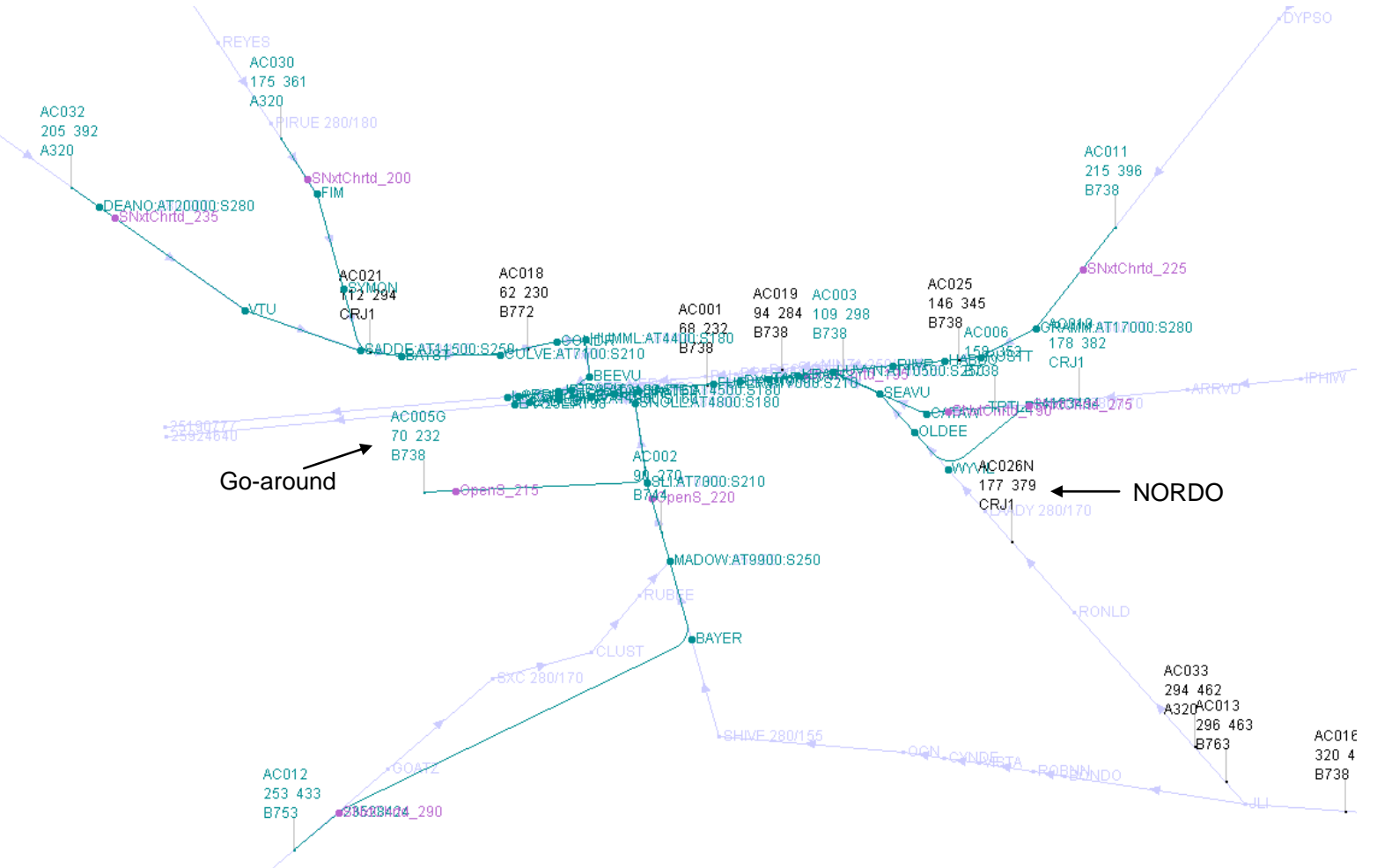

Figure 16. Trial-plan trajectories produced by the TRAC auto-planning process.

aircraft not capable of accepting trajectory modifications) are first set equal to their ETAs. Then, for each aircraft in the block to be rescheduled, the algorithm attempts to assign a suitable STA and generate a trajectory to achieve it. If there are aircraft behind the current aircraft that cannot be delayed sufficiently to meet their current STA, the algorithm first attempts to swap or advance the STA of the current aircraft. Failing that, the algorithm queries the trajectory-option generator to find an trajectory option that meets its STA, or one for which a minimum of additional delay will be needed.

The 'swap-or-advance' procedure first tests whether there is slack in front of the current aircraft. If there is, it sets the aircraft's STA earlier to remove the slack, then incrementally increases the STA until a suitable trajectory option is found. The same process is also performed for the current aircraft's trail aircraft, and for placing the current aircraft behind the (former) trail aircraft. The process results in an adjusted STA and trajectory option for which a minimum of additional delay would need to be applied later to meet the new STA.

To test the auto-planning algorithm, ten off-nominal traffic scenarios were constructed by randomly generating aircraft of various types, assigning them to RNAV arrival routes at LAX, then applying the techniques described in Section III. Each scenario includes a go-around aircraft returning to either runway 24R or 25L at LAX; the flow to the other runway includes a NORDO aircraft whose trajectory cannot be modified. The auto-planning algorithm was then invoked on the schedules for both runways in each scenario to yield the results shown in Table 1 (in the current implementation, the algorithm treats each schedule independently and rejects trajectory options that require runway reassignments).

In all cases the algorithm generated recovery plans with schedules that were either completely within tolerances, or for which a minimum of additional delay was required. The smallest processing times were produced for solutions achievable with speed changes alone. Seventeen recovery plans were produced in about one minute or less, with most processing times around $40 \mathrm{~s}$. Cases in which the swap-or-advance procedure was invoked took an unacceptably long time to process, largely due to the one-second increments used to reposition STAs. In most cases the resulting schedules had less total delay than the initial schedules (shown as positive 'delay reduction' values in Table 1), though in some cases the amount of delay was increased. These cases typically involved aircraft that were slightly behind schedule that were modified by the algorithm to instead require some additional delay. The algorithm currently does not produce vectoring options, and does not check that the resulting trajectories are conflict-free.

10

American Institute of Aeronautics and Astronautics 
Table 1. Results of applying auto-plan process to ten traffic scenarios with off-nominal situations.

\begin{tabular}{|c|c|c|c|c|c|c|}
\hline Scenario & $\begin{array}{c}\text { Scheduling } \\
\text { Point }\end{array}$ & $\begin{array}{c}\text { Off- } \\
\text { Nominal }\end{array}$ & $\begin{array}{l}\text { \# Aircraft } \\
\text { to Plan }\end{array}$ & $\begin{array}{l}\text { Time to } \\
\text { Plan (s)* }\end{array}$ & $\begin{array}{c}\text { Delay } \\
\text { Reduction (s) }\end{array}$ & Notes \\
\hline \multirow{2}{*}{1} & LAX24R & Go-Around & 3 & 38.3 & 253.5 & $\begin{array}{l}\text { Base extension for go-around; } \\
\text { speeds for straight-ins }\end{array}$ \\
\hline & LAX25L & NORDO & 4 & 38.8 & $147.3^{\dagger}$ & $\begin{array}{l}\text { Direct-to’s plus fast speeds to } \\
\text { advance }\end{array}$ \\
\hline \multirow[t]{2}{*}{2} & LAX24R & NORDO & 4 & 36.6 & 126.4 & $\begin{array}{l}\text { Slight additional delay needed for } \\
\text { aircraft ahead of NORDO }\end{array}$ \\
\hline & LAX25L & Go-Around & 3 & 34.7 & 138.1 & One direct-to plus fast speed \\
\hline \multirow[t]{2}{*}{3} & LAX24R & Go-Around & 3 & 36.1 & -102.2 & $\begin{array}{l}\text { Speed for go-around; required slight } \\
\text { advance became } \sim 50 \text { s delay }\end{array}$ \\
\hline & LAX25L & NORDO & 5 & 20.9 & -4.5 & All speed changes \\
\hline \multirow{2}{*}{4} & LAX24R & NORDO & 2 & 24.4 & -52.4 & $\begin{array}{l}\text { Fast speed for aircraft ahead of } \\
\text { NORDO }\end{array}$ \\
\hline & LAX25L & Go-Around & 5 & 29.1 & 94.7 & Within-tolerance using speeds alone \\
\hline \multirow[t]{2}{*}{5} & LAX24R & Go-Around & 7 & 39.5 & 99.5 & $\begin{array}{l}\text { Base-extension, direct-to plus } \\
\text { speeds }\end{array}$ \\
\hline & LAX25L & NORDO & 6 & 61.7 & 1.9 & Some paths plus speeds \\
\hline \multirow{2}{*}{6} & LAX24R & NORDO & 2 & 31.8 & 36.6 & All speed changes \\
\hline & LAX25L & Go-Around & 7 & 41.0 & 174.9 & Paths plus speeds \\
\hline \multirow{2}{*}{7} & LAX24R & Go-Around & 5 & 37.6 & 234.2 & Path option like CMS transition path \\
\hline & LAX25L & NORDO & 8 & 281.3 & -18.6 & Attempted swaps slow processing \\
\hline \multirow[t]{2}{*}{8} & LAX24R & NORDO & 2 & 41.0 & 46.3 & $\begin{array}{l}\text { Required slight advance became } \\
\sim 50 \text { s delay }\end{array}$ \\
\hline & LAX25L & Go-Around & 4 & 267.2 & 49.5 & Attempted swaps slow processing \\
\hline \multirow{2}{*}{9} & LAX24R & Go-Around & 4 & 40.1 & 138.9 & Speeds plus base extension \\
\hline & LAX25L & NORDO & 5 & 27.9 & -33.0 & All speed changes \\
\hline \multirow[t]{2}{*}{10} & LAX24R & NORDO & 6 & 46.1 & 211.8 & $\begin{array}{l}\text { Multiple base extensions with } \\
\text { speeds }\end{array}$ \\
\hline & LAX25L & Go-Around & 8 & 261.8 & 278.8 & Attempted swaps slow processing \\
\hline
\end{tabular}

*2.3 GHz dual-core Dell D620 laptop, 4 GB RAM ${ }^{\dagger}$ Also reduced schedule slack $9.5 \mathrm{~s}$

Most of the solutions were, however, largely conflict free; in practice, when conflicts remain a controller might make a small change to the solution, such as substituting vectors that yield approximately the same amount of delay as a speed-change trajectory.

\section{Conclusion}

This paper presents research aimed at using TRAC to model off-nominal situations and investigate recovery plans. Methods for constructing off-nominal situations, generating trajectory options, and formulating recovery plans were implemented and tested. The results indicate that in most cases reasonable solutions can be produced in an acceptably short time. Because contextual factors exist that are not captured in the auto-planning problem formulation, a traffic management supervisor will need to assess any auto-generated plan; the TRAC implementation allows for this by displaying all proposed trajectory modifications.

The research represents a first step toward a potential controller tool for supporting robust schedule-based terminal-area arrival management. Additional research on metrics for assessing the relative merit of a particular schedule is needed, as are improvements to the trajectory-generation and auto-planning processes. First, detailed trade studies of suitable parameter values should be undertaken. Second, the TRAC conflict-probing functionality should be integrated with the auto-planning process to produce conflict-free trajectories, or at least flag those trajectories that may have conflicts. Third, it could be useful to enable more interactivity with the recovery plan to, for example, allow the supervisor to examine alternate trajectory solutions for specific aircraft, or select a subset of trajectories to implement at the current time. Issues related to distributing relevant portions of a plan for inspection 
by sector controllers should also be considered. Future human-in-the-loop research could include testing of a refined tool.

\section{Acknowledgments}

This research was supported by the NASA Airspace Systems Super-Density Operations research focus area, (Shannon Zelinski, Associate Principle Investigator), and benefited from discussions with Dr. Everett Palmer, Michael Kupfer, and Joey Mercer.

\section{References}

${ }^{1}$ Joint Planning and Development Office, “Concept of Operations for the Next Generation Air Transportation System, Version 3.2," September 30, 2010.

${ }^{2}$ Swenson, H., Thippavong, J., Sadovsky, A., Chen, L., Sullivan, C., and Martin, L., "Design and Evaluation of the Terminal Area Precision Scheduling and Spacing System," Proceedings of the Ninth USA/Europe Air Traffic Management Research and Development Seminar, Berlin, 2010.

${ }^{3}$ Kupfer, M., Callantine, T., Martin, L., Mercer, J., and Palmer, E., “Controller Support Tools for Schedule-Based TerminalArea Operations," Proceedings of the Ninth USA/Europe Air Traffic Management Research and Development Seminar, Berlin, 2010.

${ }^{4}$ Callantine, T., Cabrall, C., Kupfer, M., Martin, L., Mercer, J., and Palmer, E., "Investigating the Impact of Off-Nominal Events on High-Density 'Green’ Arrivals,” Proceedings of the Thirtieth Digital Avionics Systems Conference, Seattle, October, to appear.

${ }^{5}$ Callantine, T., “An Integrated Tool for NextGen Concept Design, Fast-Time Simulation, and Analysis,” Proceedings of the AIAA Modeling and Simulation Technologies Conference, Honolulu, HI, AIAA-2008-6343, 2008.

${ }^{6}$ Callantine, T. “TRAC Trial Planning and Scenario Generation to Support Super-Density Operations Studies,” Proceeedings of the AIAA Modeling and Simulation Technologies Conference, Chicago, IL, AIAA-2009-5836, 2009.’

${ }^{7}$ Ren, L., and Clarke, J-P., "Separation Analysis Methodology for Designing Area Navigation Procedures," Journal of Guidance, Control, and Dynamics, Vol. 30, No. 5, 2007, pp. 277-297.

${ }^{8}$ Callantine, T., and Palmer, E., "Fast-Time Simulation Studies of Terminal-Area Spacing and Merging Concepts," Proceedings of the $22^{\text {nd }}$ Digital Avionics Systems Conference, Indianapolis, 2003, IEEE: 03CH37449 [CD-ROM].

${ }^{9}$ Callantine, T., "Graphical Specification of Trajectory-Modification Options in TRAC," Proceedings of the AIAA Modeling and Simulation Technologies Conference, Toronto, Ontario, AIAA-2010-8634, 2010.

${ }^{10}$ Prevot, T., Lee, P., Callantine, T., Mercer, J., Homola, J., Smith, N., and Palmer, E., "Human-in-the-Loop Simulation of NextGen Concepts in the Airspace Operations Laboratory," Proceedings of the AIAA Modeling and Simulation Technologies Conference, Toronto, Ontario, AIAA-2010-7609, 2010.

${ }^{11}$ Krozel, J., Penny, S., Prete, J., and Mitchell, J., “Automated Route Generation for Avoiding Deterministic Weather in Congested Airspace,” Journal of Guidance, Control, and Dynamics, Vol. 30, No. 1, 2007, pp. 144-153.

${ }^{12}$ Roy, K., Bayen, A., and Tomlin, C., "Polynomial Time Algorithms for Scheduling of Arrival Aircraft," Proceedings of the AIAA Guidance, Navigation, and Control Conference, San Francisco, AIAA-2005-6044, 2005. 